\title{
Chylous ascites following repair of total anomalous pulmonary venous connection coexisting with a persistent left superior vena cava in a neonate: a case report
}

\author{
Wen Zeng ${ }^{1 \wedge}$, Yue $\mathrm{Hu}^{2}$, Jun Feng ${ }^{2}$, Xiaoli $\mathrm{Luo}^{3} \wedge$ \\ ${ }^{1}$ Department of Neonatology, Chengdu Women's and Children's Central Hospital, School of Medicine, University of Electronic Science and \\ Technology of China, Chengdu, China; '2Surgical Intensive Care Unit, Chengdu Women's and Children's Central Hospital, School of Medicine, \\ University of Electronic Science and Technology of China, Chengdu, China; ${ }^{3}$ Department of Pediatric Critical Medicine, Chengdu Women's and \\ Children's Central Hospital, School of Medicine, University of Electronic Science and Technology of China, Chengdu, China \\ Correspondence to: Xiaoli Luo. Department of Pediatric Critical Medicine, Chengdu Women's and Children's Central Hospital, 1617 Riyue Avenue, \\ Qingyang District, Chengdu 611731, China. Email: 55050625@qq.com.
}

\begin{abstract}
Chylous ascites refers to the accumulation of lymphatic fluid in the peritoneal cavity. The causes of chylous ascites are various, and commonly include traumatic injury and obstruction, which disrupt the lymphatic system. In addition, cardiothoracic surgery may injure the thoracic duct and lead to chylothorax. However, there are very few reported cases of isolated chylous ascites developing following cardiothoracic surgery. In this paper, we report a case of postoperative chylous ascites in a full-term neonate. The infant underwent cardiothoracic surgery via thoracotomy to repair total anomalous pulmonary venous connection coexisting with a persistent left superior vena cava on day of life 17 , and there was a significant increase in abdominal girth on postoperative day 12 (day of life 29). Abdominal ultrasound revealed an $8 \mathrm{~mm}$ thick ascites without pleural effusion. Abdominal paracentesis was performed and the milky-white peritoneal fluid was positive for Sudan III staining and the chylous test. The triglyceride concentration of the ascitic fluid was $691 \mathrm{mg} / \mathrm{dL}$ and the concentration of protein was $39.4 \mathrm{~g} / \mathrm{L}$. Additionally, the ascitic fluid also contained $6360 \times 10^{6} / \mathrm{L}$ of white blood cells, predominantly lymphocytes. These results suggested the infant developed chylous ascites. Conservative management with fasting and medium-chain triglycerides-based formula successfully resolved the chylous ascites without reoccurrence. We present our experience of this rare condition and discuss the possible causes of chylous ascites in this case.
\end{abstract}

Keywords: Chylous ascites; heart defects; surgery; neonate; case report

Submitted Aug 23, 2020. Accepted for publication Nov 30, 2020.

doi: $10.21037 /$ tp-20-258

View this article at: http://dx.doi.org/10.21037/tp-20-258

\section{Introduction}

Chylous ascites refers to the accumulation of lymphatic fluid within the peritoneal cavity as a result of various underlying pathologies (1). Abdominal malignancy, cirrhosis, and tuberculosis are the most common causes of chylous ascites in adults, while lymphatic anomaly is the most frequent atraumatic cause in children. In addition, surgical interventions such as abdominal aneurysm repair, retroperitoneal lymphadenectomy, placement of peritoneal dialysis catheter, and inferior vena cava resection can also lead to postoperative chylous ascites (2).

In this report, we describe a unique case of neonatal

^ ORCID of Wen Zeng: 0000-0002-8064-3203; Xiaoli Luo: 0000-0002-0002-944X. 

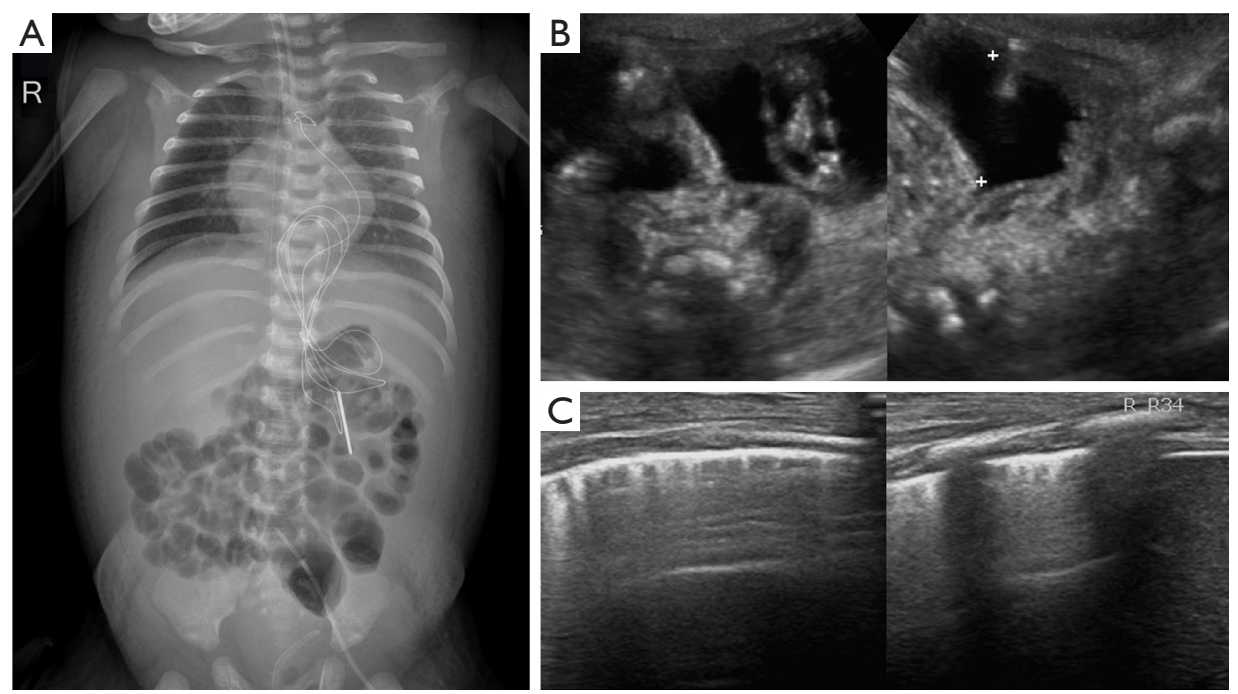

Figure $1 \mathrm{X}$-ray and abdominal and pulmonary ultrasound images on postoperative day 12 (DOL 29). (A) Combined chest and abdominal X-ray revealed centralized gas-filled bowel loops and no pleural effusion; (B) a large volume of ascites was identified by abdominal ultrasound; (C) no pleural effusion was identified by pulmonary ultrasound.

chylous ascites following repair of total anomalous pulmonary venous connection (TAPVC) and a persistent left superior vena cava (PLSVC). This condition has not been described previously. We present the following case in accordance with the Case Reports (CARE) reporting checklist (available at http://dx.doi.org/10.21037/tp20-258).

\section{Case presentation}

The patient was a female infant born at 40 weeks' gestation (weighing 3,180 g) to a 26-year-old gravida 2, para 1 mother, via normal spontaneous vaginal delivery. Prenatal laboratory tests yielded unremarkable results. Her Apgar scores were 10, 10, and 10 at 1, 5, and 10 minutes, respectively. Around 20 minutes after birth, the neonate was noted to have tachypnea with groan and cyanosis. Oxygen at 5 liters per minute via hood and empirical antibiotics were administered. On day of life (DOL) 9, the infant presented with significantly increased work of breathing and an oxygen saturation of $80 \%$ on pulse oximetry. She was intubated and placed on a conventional ventilator, and was subsequently transferred to our institution for further evaluation and management. A transthoracic echocardiogram showed cardiac total anomalous pulmonary venous connection (TAPVC) combined with an atrial septal defect (ASD) (8 $\mathrm{mm}$ in diameter). A computed tomography angiography (CTA) was performed to define the pulmonary venous anatomy, and confirmed cardiac TAPVC, persistent left superior vena cava (PLSVC), and ASD. The infant underwent surgical repair via thoracotomy on DOL 17 . Intraoperative findings showed that the pulmonary veins formed a common pulmonary vein (CPV) and then connected to the dilated right atrium (RA), coexisting with an ASD (4 $\mathrm{mm}$ in diameter) and PLSVC connected to the left atrium. During surgery, the cardiac TAPVC was corrected, the PLSVC was ligated, and the ASD was closed.

On the postoperative day 2 (DOL 19), enteral nutrition (EN) was used in combination with parenteral nutrition (PN). Complete EN with expressed breast milk was given on postoperative day 8 (DOL 25). On postoperative day 10 (DOL 27), the infant developed edema of the face and abdominal wall with normal levels of serum albumin. On the postoperative day 12 (DOL 29), there was a significant increase in abdominal girth. The chest and abdominal $\mathrm{X}$-ray revealed an opacity in the right upper lung field and a distended abdomen with centralized bowel loops (Figure 1A). Abdominal ultrasound revealed $8 \mathrm{~mm}$ thick ascites (Figure 1B) without pleural effusion (Figure 1C).

Given these findings, abdominal paracentesis was performed and yielded $130 \mathrm{~mL}$ of milky-white fluid (Figure 2). The biochemical characteristics of the peritoneal fluid sample were as follows: glucose $5.51 \mathrm{mmol} / \mathrm{L}$, protein $39.4 \mathrm{~g} / \mathrm{L}$, lactate dehydrogenase (LDH) $173.9 \mathrm{U} / \mathrm{L}$, 


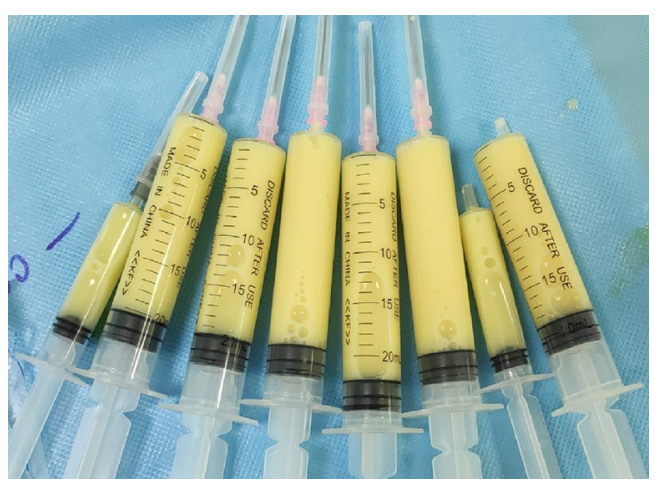

Figure 2 Milky-white fluid extracted by paracentesis on postoperative day 12 (DOL 29).

and adenosine deaminase (ADA) 4.6 U/L. The fluid contained $6,360 \times 10^{6} / \mathrm{L}$ of white blood cells consisting of $15 \%$ neutrophils and $60.7 \%$ lymphocytes. The fluid was positive for Sudan III staining and the chylous test. The triglyceride concentration of the ascitic fluid was $691 \mathrm{mg} / \mathrm{dL}$, and microbiology cultures were negative. These results suggested the infant developed chylous ascites. Breastfeeding was suspended and was switched to total parenteral nutrition (TPN) without lipid emulsion supply. During the next 2 days (DOL 30 and DOL 31), a total of $110 \mathrm{~mL}$ of milky-white fluid was found in the drain. On postoperative day 15 (DOL 32), the drain fluid decreased to $45 \mathrm{~mL}$. The infant's diet was changed to a medium-chain triglyceride (MCT)-based formula after 5 days of fasting (DOL 34). Abdominal ultrasound showed that the ascites decreased to $1.8 \mathrm{~mm}$ thick on DOL 33, and no ascites were found on the postoperative day 26 (DOL 43) (Figure 3). The infant's nutrition was switched to breast milk and normal formula on postoperative day 26 (DOL 44). There was no re-accumulation of ascites until discharge. The infant has been followed-up for more than 3 months, and there has been no sign of abdominal distension or recurrent ascites.

All procedures performed in studies involving human participants were in accordance with the ethical standards of the institutional and/or national research committee(s) and with the Helsinki Declaration (as revised in 2013). Written informed consent was obtained from the patient.

\section{Discussion}

Chylous ascites is a rare form of ascites $(<1 \%)$ caused by leakage and accumulation of lipid-rich lymph within the peritoneal cavity (1). Its etiology varies across different age groups. In newborn and young children, the most common etiology is lymphatic anomalies, whereas in adults, abdominal malignancy is the most common cause $(1,2)$. Chylous ascites can also occur as an infrequent complication following intra-abdominal surgery, which can include abdominal aneurysm repair, retroperitoneal lymphadenectomy, placement of peritoneal dialysis catheter, liver transplantation, and inferior vena cava resection (2). Symptoms of chylous ascites are related to the amount of accumulated fluid $(1,3)$. Painless abdominal distention is the most frequent symptom. With increased ascites and abdominal pressure, a patient may experience dyspnea (shortness of breath). The diagnosis of chylous ascites is based on the character and composition of the ascitic fluid, and therefore, paracentesis is the most important diagnostic tool. Triglyceride levels of $>200 \mathrm{mg} / \mathrm{dL}$ in ascitic fluid is used to diagnose chylous ascites $(1,4)$.

In this report, we describe the development of chylous ascites in a neonate following repair of TAPVC and PLSVC. The exact cause of the chylous ascites in this case could not be determined. Several possible mechanisms are discussed below. As mentioned above, lymphatic anomalies are the most common cause of chylous ascites in neonatal infants. In our case, there was no solid evidence to rule out the possibility of lymphatic anomalies because a lymphangiography was not performed. However, conservative management resolved the chylous ascites in a short period of time without reoccurrence. We assume the possibility of lymphatic anomalies in this case was relatively low.

The thoracic duct is prone to injury during cardiothoracic surgical procedures. Injury to the thoracic duct may cause leakage of chyle, which would most likely be associated with the development of a left chylothorax (5). In this case, isolated chylous ascites occurred after cardiothoracic surgery, while no pleura effusion was detected. It appears unlikely that the chylous ascites in our patient was related to traumatic injury of the thoracic duct during surgery. However, it is worth noting that obstruction of the thoracic portion of the thoracic duct either intraor postoperatively may increase the pressure in the major lacteal within the mesentery and intestinal wall, cause exudation and leakage of chyle, and subsequently result in the development of chylous ascites $(6,7)$. This was a possible pathogenic factor for chylous ascites in our patient.

In this case, the newborn was diagnosed with cardiac TAPVC with a PLSVC, which is an extremely rare combination. TAPVC refers to a congenital heart disease in 

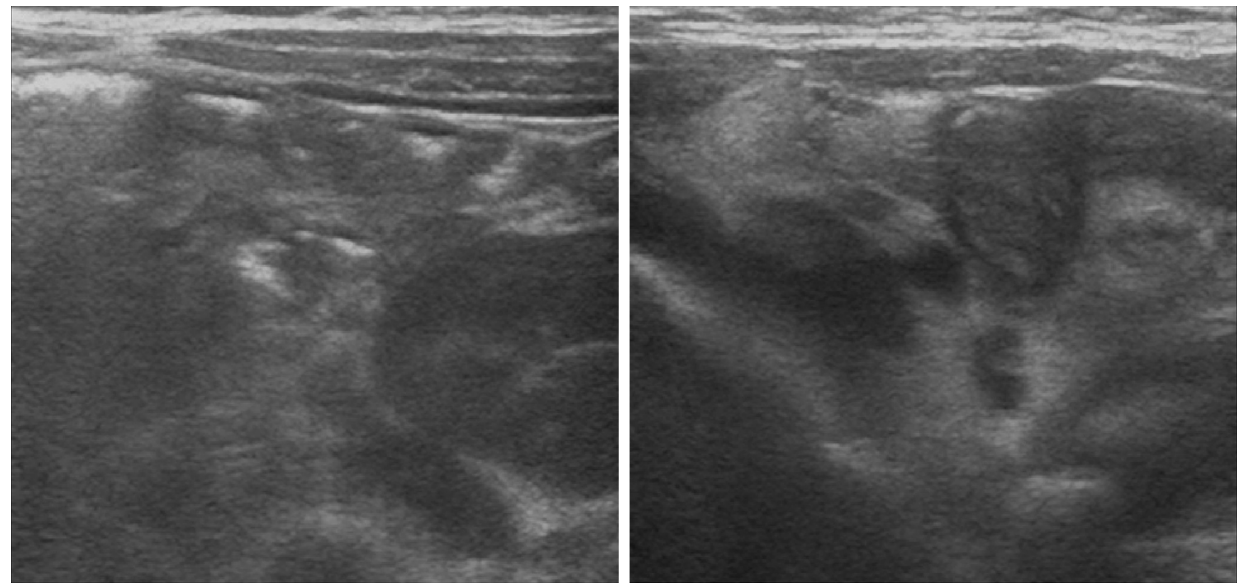

Figure 3 No ascites was identified by abdominal ultrasound on the postoperative day 26 (DOL 43).

which all pulmonary veins do not return to the left atrium, but instead connect to the systemic veins, right atrium, or coronary sinus (8). The pulmonary veins in our patient formed a CPV and were connected to the right atrium, resulting in an overload of right heart and pulmonary hypertension. PLSVC of this infant drained directly into the left atrium with a $4 \mathrm{~mm}$ ASD. PLSVC draining into the left atrium is a relatively rare condition, and severe cases can potentially lead to pulmonary hypertension and heart failure. This neonate had congenital heart defects, which could have been a pathological cause of the chylous ascites. Previous studies have reported that chylous ascites is caused by pulmonary hypertension, ventricular septal defect, and constrictive pericarditis (9-11), indicating that chylous ascites is related to heart disease. High venous pressure increases lymphatic pressure and lymph flow, resulting in more lymphatic production. However, the venolymphatic junction restricts lymph return to the central veins, leading to an increase in lymphatic pressure (9). This increased intestinal lymphatic pressure may cause localized rupture of the lymphatics, resulting in chylous ascites. In the present case, the infant had facial and abdominal edema prior to detection of the ascites, which might have been a clue regarding the increased venous pressure.

Dietary management is the first option for the conservative management of chylous ascites $(5,12)$. A MCTbased diet is recommended. MCTs are absorbed directly into intestinal cells and enter the portal venous system (6). Thus, a low-fat diet with MCT supplementation reduces chyle production in the peritoneal fluid. However, the use of MCT-based infant formula should be limited to
3-4 months as long-chain triglycerides (LCT) are required for central nervous system development (13). TPN and fasting are the other conservative treatment options for chylous ascites. During the period of TPN and fasting, intestinal lymph flow is reduced, which aids in the repair of the lymphatic system (12).

Paracentesis is not only a diagnostic tool, but also a therapeutic measure. For patients with large-volume chylous ascites, repeated paracentesis and continuous peritoneal drainage help to temporarily relieve abdominal distention and dyspnea. However, the major drawbacks include hypoproteinemia, electrolyte imbalance, decrease of immunoglobulins, and increased risk of infection $(14,15)$. It is also important to mention that paracentesis should always be combined with other conservative measures.

In addition to this, somatostatin can reduce the production of lymph. Octreotide, as a synthetic longacting somatostatin analogue, can bind to the somatostatin receptor and decrease lymphorrhea $(1,15)$. In some persistent, refractory cases, somatostatin/octreotide combined with TPN is a therapeutic strategy to close lymphatic leakage or rapidly relieve symptoms. There have been reports of the successful use of somatostatin/octreotide for chylothorax and chylous ascites in neonates $(3,15,16)$. However, somatostatin/octreotide may interrupt the insulin/ glucagon balance and suppress the thyroid-stimulating hormone at the hypothalamus/pituitary level (17). Furthermore, there are no specific guidelines for the use of somatostatin/octreotide in neonates, although some reports indicate that octreotide is safe for neonatal use $(18,19)$. However, more data regarding the optimal initial dosing, 
duration, safety, and efficacy are required.

Surgical intervention should be considered in patients when 3-4 weeks of conservative management fails $(2,12)$. If chylous ascites recurs after resumption of a normal diet, surgical exploration may be required. Prior to surgery, it is suggested that lymphangiography or lymphoscintigraphy be performed to help identify the site of leakage or the presence of a fistula.

\section{Conclusions}

Chylous ascites is a rare clinical complication following cardiothoracic surgery, which can occur as a result of intraoperative lymphatic system disruption. It should also be considered as a possibility when there is progressive abdominal distention. X-ray and ultrasound of the abdomen are used to confirm the diagnosis of ascites. Paracentesis should be performed, and triglyceride levels of $>200 \mathrm{mg} / \mathrm{dL}$ in ascitic fluid should be considered the diagnostic criteria for chylous ascites. Treatment of chylous ascites is usually conservative, and can include TPN or enteral diet with MCT applied for several weeks, although some refractory cases require surgical treatment.

\section{Acknowledgments}

Funding: None.

\section{Footnote}

Reporting Checklist: The authors have completed the CARE reporting checklist. Available at http://dx.doi.org/10.21037/ tp-20-258

Conflicts of Interest: All authors have completed the ICMJE uniform disclosure form (available at http://dx.doi. org/10.21037/tp-20-258). The authors have no conflicts of interest to declare.

Ethical Statement: The authors are accountable for all aspects of the work in ensuring that questions related to the accuracy or integrity of any part of the work are appropriately investigated and resolved. All procedures performed in studies involving human participants were in accordance with the ethical standards of the institutional and/or national research committee(s) and with the Helsinki Declaration (as revised in 2013). Written informed consent was obtained from the patient.
Open Access Statement: This is an Open Access article distributed in accordance with the Creative Commons Attribution-NonCommercial-NoDerivs 4.0 International License (CC BY-NC-ND 4.0), which permits the noncommercial replication and distribution of the article with the strict proviso that no changes or edits are made and the original work is properly cited (including links to both the formal publication through the relevant DOI and the license). See: https://creativecommons.org/licenses/by-nc-nd/4.0/.

\section{References}

1. Bhardwaj R, Vaziri H, Gautam A, et al. Chylous ascites: a review of pathogenesis, diagnosis and treatment. J Clin Transl Hepatol 2018;6:105-13.

2. Kostov S, Yordanov A, Slavchev S, et al. First case of chylous ascites after laparoscopic myomectomy: a case report with a literature review. Medicina (Kaunas) 2019;55:624.

3. Bulbul A, Okan F, Nuhoglu A. Idiopathic congenital chylothorax presented with severe hydrops and treated with octreotide in term newborn. J Matern Fetal Neonatal Med 2009;22:1197-200.

4. Thaler MA, Bietenbeck A, Schulz C, et al. Establishment of triglyceride cut-off values to detect chylous ascites and pleural effusions. Clin Biochem 2017;50:134-8.

5. Bender B, Murthy V, Chamberlain RS. The changing management of chylothorax in the modern era. Eur J Cardiothorac Surg 2016;49:18-24.

6. Almakdisi T, Massoud S, Makdisi G. Lymphomas and chylous ascites: review of the literature. Oncologist 2005;10:632-5.

7. Sy ED, Lin CH, Shan YS, et al. Chyloperitoneum: a postoperative complication after repair of tracheoesophageal fistula. J Pediatr Surg 2001;36:E1.

8. Files MD, Morray B. Total anomalous pulmonary venous connection: preoperative anatomy, physiology, imaging, and interventional management of postoperative pulmonary venous obstruction. Semin Cardiothorac Vasc Anesth 2017;21:123-31.

9. Ridruejo E, Mandó OG. Chylous ascites as the main manifestation of left ventricular dysfunction: a case report. BMC Gastroenterol 2005;5:25.

10. Petrasek AJ, Ameli FM. Conservative management of chylous ascites complicating aortic surgery: a case report. Can J Surg 1996;39:499-501.

11. Ootaki Y, Verghese GR, Ungerleider RM. Development of chylous ascites in an infant with ventricular septal defect: a 
case report. Cardiol Young 2016;26:1225-7.

12. Karagol BS, Zenciroglu A, Gokce S, et al. Therapeutic management of neonatal chylous ascites: report of a case and review of the literature. Acta Paediatr 2010;99:1307-10.

13. Chye JK, Lim CT, Van der Heuvel M. Neonatal chylous ascites--report of three cases and review of the literature. Pediatr Surg Int 1997;12:296-8.

14. Wasmuth-Pietzuch A, Hansmann M, Bartmann P, et al. Congenital chylothorax: lymphopenia and high risk of neonatal infections. Acta Paediatr 2004;93:220-4.

15. Andreou A, Papouli M, Papavasiliou V, et al. Postoperative chylous ascites in a neonate treated successfully with octreotide: bile sludge and cholestasis. Am J Perinatol 2005;22:401-4.

Cite this article as: Zeng W, Hu Y, Feng J, Luo X. Chylous ascites following repair of total anomalous pulmonary venous connection coexisting with a persistent left superior vena cava in a neonate: a case report. Transl Pediatr 2021;10(1):188-193. doi: $10.21037 /$ tp-20-258
16. Olivieri C, Nanni L, Masini L, et al. Successful management of congenital chylous ascites with early octreotide and total parenteral nutrition in a newborn. BMJ Case Rep 2012;2012:bcr2012006196.

17. Zaki SA, Krishnamurthy MB, Malhotra A. Octreotide use in neonates: a case series. Drugs R D 2018;18:191-8.

18. Sooklin L, Anand AJ, Rajadurai VS, et al. Management of large congenital chylous ascites in a preterm infant: fetal and neonatal interventions. BMJ Case Rep 2020;13:e235849.

19. Chandran S, Agarwal A, Llanora GV, et al. Necrotising enterocolitis in a newborn infant treated with octreotide for chylous effusion: is octreotide safe? BMJ Case Rep 2020;13:e232062. 